Memoirs of the College of Science, University of Kyoto, Series A

Vol. XXXIII, Mathematics No. 3, 1961.

\title{
Additive functionals of the Brownian path
}

\author{
By
}

H. P. McKean, JR. and Hiroshi TANAKA

(Communicated by Prof. K. Itô, March 14, 1961)

\section{INTRODUCTION.}

A functional $\mathrm{f}(t)=\mathrm{f}(t, w)$ of $t(\geq 0)$ and a several-dimensional Brownian path $w: t \rightarrow x(t)$ is said to be additive if

$1.1 \mathrm{f}(t, w)$ depends upon $t$ and $x(s): s \leq t$ alone.

1.2 $0=\mathrm{f}(0) \leq \mathrm{f}<+\infty$

1. $3 \mathrm{f}(t \pm)=\mathrm{f}(t)$

1. $4 \mathrm{f}(t)=\mathrm{f}(s)+\mathrm{f}\left(t-s, w_{s}^{+}\right) \quad t \geq s$,

where $w_{s}^{+}$is the shifted path $w_{s}^{+}: t \rightarrow x(t+s)$; for example, $\mathrm{f}(t)=\int_{0}^{t} f(x(s)) d s$ is an additive functional if $f \geq 0$ is bounded and Borel.

K. Itô and H. P. McKean, Jr. [13] proved that in the 1-dimensional case such an additive functional is an integral

$1.5 \quad \mathrm{f}(t)=\int \mathrm{t}(t, b) e(d b)$

of the standard Brownian local times

$1.6 \mathrm{t}(t, a)=\lim _{b \downarrow a} \frac{\text { measure }(s: a \leq x(s)<b, s \leq t)}{b-a}$

with respect to a non-negative measure $e$, finite on bounded intervals.

Brownian local times are not available in $d \geq 2$ dimensions, but $f$ can still be expressed as a (formal) Hellinger integral

$1.7 \quad \mathrm{f}(t)=\int \frac{\text { measure }(s: x(s) \in d b, s \leq t) e(d b)}{d b}$

with a non-negative measure $e$ which is smooth in the sense that 
each bounded open $D$ is the union of an increasing series of sets $B_{n}(n \geq 1)$, closed in $D$, such that

1. 8a the charge distributions $e \mid B_{n}$ have bounded potentials $\int_{B_{n}} G d e$ $\leq n(n \geq 1)$, where $G$ is the Green function of $D$;

$1.8 \mathrm{~b}$ for large $n$, depending upon the path, the Brownian particle lies in $B_{n}$ until it leaves $D$, i.e.,

$$
P \cdot\left[x(t) \in B_{n}, t<\min (s: x(s) \notin D), n \uparrow+\infty\right]=1,
$$

where $P .(B)$ is the probability of the event $B$ as a function of the starting point of the Brownian motion.

The correspondence embodied in 1.7 between the class of smooth measures $e$ and the class of additive functionals $f$ is one to one and onto.

1. 8a implies that $e(B)=0$ unless $B$ has positive logarithmic capacity in the 2-dimensional case or has positive Newtonian capacity in the $d \geq 3$ dimensional case; thus, a smooth measure cannot attach positive mass to a line in 3 dimensions, nor, in 4 dimensions, to a surface. But it can be singular relative to Lebesgue measure; the simplest example in 3 dimensions is the uniform distribution on the spherical surface $|a|=1$.

Choose $d=3, e(d b)=|b|^{a} d b$, and let $G$ be the Green function of $D:|a|<1$; then

1.9a $p=\int G d e$

$$
\begin{array}{ll}
\leq \text { constant }(2+\alpha)^{-1} & \alpha>-2 \\
<+\infty \text { except at } 0 & -3<\alpha \leq-2 \\
\equiv+\infty & \alpha \leq-3,
\end{array}
$$

$1.9 \mathrm{~b} \quad \mathfrak{F}(e) \equiv \iint G d e d e<+\infty \quad \alpha>-5 / 2$,

and it follows that $e$ is smooth for $\alpha>-2$. $e$ is not smooth for $\alpha \leq-2$; in fact, choosing $B_{n} \uparrow D$ as needed for 1.8 ,

$$
\begin{aligned}
1.10 \quad E_{0} & {\left[\int_{0}^{\varepsilon}|x(s)|^{\alpha} d s, x(t) \in B_{n}, t<\varepsilon\right] } \\
& \leq \int_{0}^{+\infty} d s \int_{B_{n}} P_{0}\left[x(s) \in d b, \max _{1 \leq s}|x(t)|<1\right]|b|^{\infty} \\
& \leq \int_{B_{n}} G(0, b) d e<+\infty,
\end{aligned}
$$


thanks to $1.8 \mathrm{a}$, and

$1.11 \lim _{\varepsilon \downarrow 0} \lim _{n \uparrow+\infty} P_{0}\left[x(t) \in B_{n}, t<\varepsilon\right]=1$

thanks to $1.8 \mathrm{~b}$, while, as is not hard to prove,

$$
P_{0}\left[\int_{0}^{\varepsilon}|x(s)|^{a} d s \equiv+\infty, \varepsilon>0\right]=1
$$

contradicting 1.10.

V. A. Volkonskii $[15,16]$ also studied additive functionals, establishing a special case of the above for a wider class of motions; the method used below is similar to his.

Given a 1-dimensional diffusion with the same hitting probabilities as the standard Brownian motion:

1.13

$$
\begin{aligned}
& P_{\xi}[\min (t: x(t)=a)<\min (t: x(t)=b)] \\
& =\frac{b-\xi}{b-a} \quad a<\xi<b,
\end{aligned}
$$

W. Feller [8] explained how to express the associated generator (B) as a differential opearator based upon a speed measure e, positive on open intervals :

$$
\begin{gathered}
1.14 \text { (S) } u=\frac{u^{+}(d a)}{e(d a)}=\lim _{b \downarrow a} \frac{u^{+}(b)-u^{+}(a)}{e(a, b)} \\
u^{+}(a)=\lim _{b \downarrow a} \frac{u(b)-u(a)}{b-a},
\end{gathered}
$$

and K. Itô and H. P. McKean, Jr. [13] found that its sample paths could be expressed as standard Brownian sample paths run with the stochastic clock $f^{-1}$ which is the inverse function of the additive functional (local time integral) $\mathrm{f}=\int \mathrm{t} d e$ associated with the speed measure.

V. A. Volkonskiî [15] also studied such time substitutions; his method is less explicit because it does not use local times but has the advantage that it can be applied in higher dimensions.

As will be explained below, a $d \geq 2$ dimensional diffusion with Brownian hitting probabilities has as its generator the closure of a differential operator 
$1.15 \quad$ Bs $u=-\frac{e^{u}(d b)}{e(d b)}$

based upon a (smooth) speed measure $e$, positive on the neighborhoods of H. Cartan's fine topology [2]; moreover, the associated motion is the standard Brownian motion run with the inverse function $\mathrm{f}^{-1}$ of the additive functional $\mathrm{f}$ associated with $e$, and this correspondence between the class of diffusions with Brownian hitting probabilities and the class of smooth measures $e$ positive on fine neighborhoods is one to one and onto.

\section{BROWNIAN MOTION.}

Choose $d \geq 2$, let $E^{d}=R^{d}$ it $d=2$, let it be the one-point compactification $R^{d}+\infty$ if $d \geq 3$, introduce the space of continuous sample paths $w:[0,+\infty) \rightarrow E^{d}$ with

$2.1 \quad w(t) \in R^{d} \quad t<\mathfrak{m}_{\infty}$

$$
=\infty \quad t \geq \mathrm{m}_{\infty},
$$

where $\mathfrak{m}_{\infty}=\mathfrak{m}_{\infty}(w) \leq+\infty$ and $\mathfrak{m}_{\infty} \equiv+\infty$ in case $d=2$, let $w(t)=$ $x(t, w)=x(t)$ as need be, note that $x(+\infty) \equiv \infty$ even if $d=2$, and, introducing the corresponding coordiante fields $\boldsymbol{B}_{t}=\boldsymbol{B}[x(s): s \leq t]$ and $\boldsymbol{B}=\boldsymbol{B}_{\infty+}$, let $P .(B)$ be the probability (Wiener measure) of the event $B \in \boldsymbol{B}$ as a function of the starting point of the $d$-dimentional Brownian motion with generator $\mathbb{S}=\frac{\partial^{2}}{\partial b_{1}^{2}}+\frac{\partial^{2}}{\partial b_{2}^{2}}+\cdots+\frac{\partial^{2}}{\partial b_{a}^{2}} \cdot{ }^{1}$

Brownian motion enthusiasts are familiar with the fact that the Brownian traveller starts afresh at a passage time; the full significance of this was explained by E. B. Dynkin [6] and G. Hunt [9] as follows.

An instant of time $0 \leq \mathrm{m} \leq+\infty$ depending upon the path is said to be a Markov time if

$2.2 \quad(w: \mathfrak{m}<t) \in B_{t} \quad t \geq 0$;

for example, the passage time $\mathfrak{m}_{Q}=\inf (t: x(t) \in Q)$ to a closed or

$1 \quad \mathbb{S} / 2$ is often used as the generator of the Brownian motion, but for our purpose it is simpler to omit the factor $1 / 2$. 
open $d$-dimensional figure $Q$ is a Markov time and so is $\mathfrak{m}=$ $\min \left(t: \int_{0}^{t} f(x(s)) d s=1\right)$ if $0<f \leq 1$ is a Borel function.

Given such a Markov time $m$, if $w_{\mathfrak{m}}^{+}$is the shifted path

$2.3 w_{\mathfrak{m}}^{+}: t \rightarrow x(t+\mathrm{m})$

and if $\boldsymbol{B}_{\mathfrak{m}+}$ is the field of events $B \in \boldsymbol{B}$ such that

$2.4 \quad B \cap(w: \mathfrak{m}<t) \in B_{t} \quad t \geq 0$,

then the Brownian particle starts afresh at time $t=\mathrm{m}$, i.e.,

$2.5 \quad P_{a}\left[w_{\mathfrak{m}}^{+} \in B \mid \boldsymbol{B}_{\mathfrak{m}+}\right]=P_{b}(B) \quad a \in E^{d}, B \in \boldsymbol{B}, b \equiv x(\mathfrak{m})^{2}$.

Blumenthal's 01 law [1]:

$2.6 \quad P .(B)={ }_{1}^{0} \quad B \in \boldsymbol{B}_{0+}=\bigcap_{\varepsilon>0} \boldsymbol{B}_{\varepsilon}$

is a special case of 2.5 .

A. R. Galmarino ${ }^{3}$ has pointed out that a non-negative Borel function $\mathrm{m}$ of the sample path is a Markov time if and only if

$2.7 \mathrm{a} \quad \mathrm{m}(u)<t$

2.7b $\quad x(u, u)=x(s, v) \quad s \leq t$

imply $\mathrm{m}(u)=\mathfrak{m}(v)$ and that an event $B \in \boldsymbol{B}$ is a member of $\boldsymbol{B}_{\mathfrak{m}+}$ if and only if 2.7 coupled with $u \in B$ implies $v \in B$. As a simple application of this test, note that $B_{\mathfrak{m}+}$ measures both $m$ and the past $x(\theta \wedge \mathfrak{m})(\theta \geq 0)^{4}$ because 2.7 implies $\theta \wedge \mathfrak{m}(u)=\theta \wedge \mathfrak{m}(v)<t$ and hence $x(\theta \wedge \mathfrak{m}(u), u)=x(\theta \wedge \mathfrak{m}(v), v)$.

Given bounded open $D \subset R^{d}$ with boundary $\partial D$, if $\mathrm{m}_{\partial D}$ is the exit time $\min (t: x(t) \in \partial D)$, then the hitting probability

$2.8 \quad h_{\partial D}(a, d b)=P_{a}\left[x\left(\mathfrak{m}_{\partial D}\right) \in d b\right] \quad a \in D, d b \in \partial D$

is the classical harmonic measure of $d b$ as viewed from the point $a$, and, if $G_{D}(a, b)$ is the classical Green function of $D$, then

$2.9 \quad E_{a}\left[\right.$ measure $\left(t: x(t) \in d b, t<\mathrm{m}_{\partial D}\right]=G_{D}(a, b) d b^{5} \quad a, b \in D$;

\footnotetext{
$2 x(\mathfrak{m}) \equiv \infty$ in case $\mathfrak{m}=+\infty ;$ it is understood that $P_{\infty}[x(t) \equiv \infty, t \geq 0]=1$.

3 private communication.

$4 a \wedge b$ is the smaller of $a$ and $b$.

$5 \quad$ E. $(f)=\int f d P$..
} 
for the proofs, see J. Doob [5] and G. Hunt [9].

G. Hunt $[10]$ has called a non-negative Borel function $p$ excessive on $D$ if

2. $10 \quad E_{a}\left[p(x(t)), t<\mathrm{m}_{\partial D}\right] \uparrow p(a) \quad t \downarrow 0, a \in D$.

An excessive function can be split into its greatest harmonic minorant $h$ plus the potential $\int G_{D} d e$ of a non-negative (Riesz) measure $e$, indeed, Hunt's excessive functions are the same as the superharmonic functions of F. Riesz [14]. J. Doob [5] proved that an excessive function is continuous on the Brownian path $\left(t<\mathrm{m}_{\partial D}\right)$ and that $a$ potential tends to 0 along the Brownian path $\left(t \uparrow \mathrm{m}_{\partial D}\right)$.

\section{THE ASSOCIATED MEASURE OF AN ADDITIVE FUNC- TIONAL.}

Consider an additive functional $f$ of the Brownian sample path as described in seition 1 , interpreting 1.1 to mean

3. $1 \mathrm{f}(t, w)$ is measurable $\boldsymbol{B}_{t}$ for each $t \geq 0$.

The purpose of this section is to associate with f a unique non-negative measure e such that, for each bounded open $D \subset R^{d}$,

3. $21-p_{\alpha}=\alpha \int G p_{\alpha} d e \quad p_{\alpha}=E \cdot\left[e^{-\alpha \uparrow\left(\mathfrak{m}_{\partial} D\right)}\right], \alpha>0$,

where $G$ is the Green funetion of $D$ and the integral is extended over $D$; it will follow from 3.2 that $e$ is smooth.

Ccnsider, for this purpose, the additive functional

3. $3 \quad \mathrm{f}_{a}(t)=\int_{0}^{t_{\wedge}{ }^{\mathrm{m} \partial D}} p_{a}(x(s)) \mathrm{f}(d s) \quad t \geq 0$,

and let us begin with the following simple lemmas:

a) $1-p_{a}$ is the potential of a non-negative measure $\alpha_{a}$.

b) $E \cdot\left[f_{a}\left(m_{\partial D}\right)\right]<+\infty$.

c) $f_{\alpha} \uparrow f$ as $\alpha \downarrow 0$.

d) $1-p_{a}=\alpha E \cdot\left[\mathrm{f}\left(\mathrm{m}_{\partial D}\right)\right]=\alpha \int G d e_{\alpha}$.

e) $E \cdot\left[{ }_{0}^{\mathfrak{m} \partial n} f(x(s)) \mathfrak{f}_{a}(d s)\right]=\int G f d e_{a}$ if $f \geq 0$ is a Borel function. 
f) $p_{\alpha}^{-1} e_{\alpha}(d b)=e(d b)$ is independent of $\alpha$ and of $D$.

g) $e$ is unique.

Because

$3.3 \quad E .\left(1-p_{\alpha}(x(t)), t<\mathrm{m}_{\partial D}\right)=E .\left(1-e^{-\alpha\left[\mathfrak{f}\left(\mathrm{m}_{\partial D}\right)-\mathrm{f}(t)\right]}, t<m_{\partial D}\right)$

$$
\uparrow 1-p_{a} \quad t \downarrow 0,
$$

$1-p_{\infty}$ is excessive; it is, in fact, a potential thanks to

3. 4

$$
\int_{\partial \dot{D}} h_{\partial \dot{D}}(a, d b)\left[1-p_{a}\right]=E_{a}\left(1-e^{-\alpha\left[\mathfrak{f}\left(\mathfrak{m}_{\partial D}\right)-\mathfrak{f}\left(\mathfrak{m}_{\partial} \dot{D}\right]\right.}\right) \downarrow 0 \quad \dot{D} \uparrow D,
$$

and this completes the proof of a). As to b), $p_{a}$ is continuous on the Brownian path because of 1 , and, since $f$ is continuous, $b$ ) follows on letting $n \uparrow+\infty$ in

$$
\begin{aligned}
& 1-p_{a}=E \cdot\left[\int_{0}^{\mathfrak{m} \partial D} e^{-\alpha\left[\mathfrak{f}\left(\mathfrak{m}_{\partial D}\right)-\mathfrak{f}(t)\right]} \mathfrak{f}(d t)\right] \\
& \geq \alpha E \cdot\left[\sum_{k 2^{-n}<\mathfrak{m}_{\partial D}} e^{-\alpha \mathfrak{f}\left(\mathfrak{m}_{\partial D}\left(w_{k^{2}}^{+} n\right), w_{k^{2}}^{+} n\right)} e^{-\alpha \mathfrak{f}\left(I_{k}\right)} \mathfrak{f}\left(I_{k}\right)\right] \\
& I_{k}=\left[(k-1) 2^{-n}, k 2^{-n}\right) \\
& =\alpha E \cdot\left[\sum_{k 2^{-n}<m_{\partial D}} p_{\alpha}\left(x\left(k 2^{-n}\right)\right) e^{-\alpha \mathfrak{f}\left(I_{k}\right)} \mathfrak{f}\left(I_{k}\right)\right] .
\end{aligned}
$$

Because of c), which is obvious,

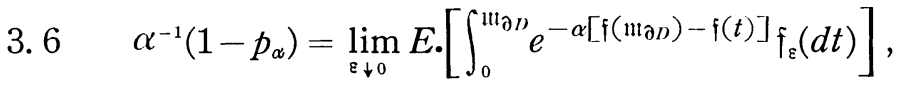

and, using the method of 3.5 and $E .\left[f_{\varepsilon}\left(\mathfrak{m}_{\partial D}\right)\right]<+\infty$, it appears that

3. 7

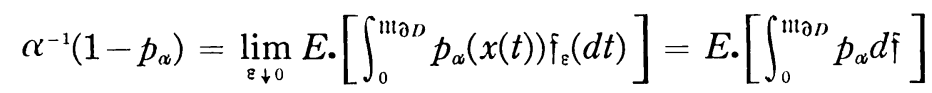

proving $\mathrm{d}$ ).

K. Itô (private communication) pointed out the following neat method for proving e). Choose closed $B \subset D$ such that $e_{\alpha}(\partial B)=0$ and let $e_{1}$ and $e_{2}$ be the charge distributions of the potentials $p_{1}=E \cdot\left[\int_{0}^{\mathfrak{m}_{\partial} D} f d \mathfrak{f}_{\alpha}\right]$ and $p_{2}=E \cdot\left[\int_{0}^{\mathfrak{m}^{\prime} D}(1-f) d \tilde{f}_{\alpha}\right]$, in which $f$ is the indicator function of $B$. Because $p_{1}$ is harmonic outside $B$ and differs from $\alpha^{-1}\left(1-p_{a}\right)=E \cdot\left[f_{\alpha}\left(m_{\partial D}\right)\right]$ by a harmonic function inside $B, e_{1}$ is 
not smaller than the restriction of $e_{\alpha}$ to $B$, and, for the same reasons, $e_{2}$ is not smaller than the restriction of $e_{a}$ to $D-B$. But $p_{1}+p_{2}=\alpha^{-1}\left(1-p_{\alpha}\right)$, whence

3. $8 p_{1}=E \cdot\left[\int_{0}^{\mathfrak{m}_{\partial} D} f d f_{\alpha}\right]=\int_{B} G d e_{\alpha}$,

and since such figures $B$ generate the class of Borel subsets of $D$, e) follows.

As to $\mathrm{f}), p_{\alpha}>0$ because $f\left(\mathrm{~m}_{\partial D}\right)<+\infty$, and, choosing $0<\beta<\alpha$, e) implies

$3.9 \int G d e_{\alpha}=E \cdot\left[\int_{0}^{m_{\partial} D} p_{\alpha} / p_{\beta} d \mathrm{f}_{\beta}\right]=\int G p_{\alpha} / p_{\beta} d e_{\beta}$,

i.e., $d e \equiv p_{\alpha}^{-1} d e_{\infty}$ is indeqendent of $\alpha$; it is also independent of $D$ because if $\dot{D} \supset D$ and if $p_{\alpha} \equiv 0$ outside $D$, then, with an obvious notation,

3.10

$$
\begin{aligned}
& \int G p_{\alpha} \dot{p}_{\beta} d e=E \cdot\left[\int_{0}^{\mathfrak{m}_{\partial} D} p_{\alpha} d \dot{\mathfrak{f}}_{\beta}\right] \\
& =E \cdot\left[\int_{0}^{\mathfrak{m}_{\partial} \dot{b}} p_{\alpha} d \dot{\mathfrak{\dagger}}_{\beta}\right]-E \cdot\left[\int_{\mathfrak{m}_{\partial D} D}^{\mathfrak{m}_{\partial} \dot{b}} p_{\infty} d \dot{\dot{\dagger}_{\beta}}\right] \\
& =\int \dot{G} p_{\alpha} \dot{p}_{\beta} d \dot{e}-\int d h_{\partial D} \int \dot{G} p_{\alpha} \dot{p}_{\beta} d \dot{e} \\
& =\int G \dot{p}_{\alpha} p_{\beta} d \dot{e} \text {. }
\end{aligned}
$$

g) is immediate from 3.2.

To establish the smoothness of $e$, take bounded open $D$ and put $B_{n}=D \bigcap\left(p_{1} \geq n^{-1}\right)$. Because $1-p_{1}$ is a potential, $B_{n}$ is closed in $D$ and increases to $D$ as $n \uparrow+\infty$; moreover, according to 3.2,

3. $11 \int_{B_{n}} G d e \leq n \int G p_{1} d e=n\left(1-p_{1}\right) \leq n$,

which is $1.8 \mathrm{a}$, and, since, along the Brownian path, $0<p_{1}$ is continuous and tends to $1\left(t \uparrow \mathrm{m}_{\partial D}\right)$,

$3.12 P \cdot\left[\inf \left(t: x(t) \notin B_{n}\right)<\mathrm{m}_{\partial D}\right]$

$$
=P \cdot\left[\inf _{t<\operatorname{nt} D} p_{1}(x(t))<n^{-1}\right] \downarrow 0 \quad n \uparrow+\infty,
$$

which verifies $1.8 \mathrm{~b}$. 


\section{UNIQUENESS.}

The following simple lemma is useful in later sections: two additive functionals $\mathfrak{f}_{1}$ and $\mathfrak{f}_{2}$ with the same bounded mean

$4.1 p=E \cdot\left[\mathrm{f}\left(\mathrm{m}_{\partial D}\right)\right]=\lim _{\varepsilon \downarrow 0} \varepsilon^{-1}\left(1-p_{\varepsilon}\right)=\int G d e \quad f=f_{1}, f_{2}$

are the same for $t \leq \mathrm{m}_{\partial D}$.

An argument similar to 3.5 implies

4.2

$$
\begin{aligned}
& E \cdot\left(\int_{0}^{m_{\partial} D}\left[f_{i}\left(m_{\partial D}\right)-f_{i}(t)\right] f_{k}(d t)\right) \\
& =E \cdot\left(\int_{0}^{\mathfrak{m} \partial \nu} p d f_{k}\right) \\
& =\int G p d e \leq\|p\|_{\infty}^{2}<+\infty \text {; }
\end{aligned}
$$

thus, putting $\mathrm{f}=\mathrm{f}_{2}-\mathrm{f}_{1}$,

$4.3 \quad E .\left[f\left(\mathrm{~m}_{\partial D}\right)^{2}\right]$

$=2 E \cdot\left[\int_{0}^{m_{\partial D}}\left[\mathrm{f}\left(\mathrm{m}_{\partial D}\right)-\mathrm{f}(t)\right] \mathrm{f}(d t)\right]$

$=0$,

and hence

$4.40=E \cdot\left[\mathrm{f}\left(\mathrm{m}_{\partial D}\right) \mid \boldsymbol{B}_{t \wedge \mathrm{m} \partial D^{+}}\right]=E \cdot\left[\mathrm{f}\left(\mathrm{m}_{\partial D}\right)-\mathrm{f}(t) \mid \boldsymbol{B}_{t \wedge \mathrm{m}_{\partial D^{+}}}\right]+\mathrm{f}(t)$

$$
=\mathrm{f}(t) \quad t \leq \mathrm{m}_{\partial D}
$$

as desired.

It is a simple matter to deduce from this that two additive functionals with the same associated measure are the same; indeed, the difference $p$ of two solutions of 3.2 satisfies $-p=\alpha \int G p d e$, which implies

$$
0 \geq-\int p^{2} d e=\mathfrak{F}(p d e)=\int G p d e p d e,
$$

and it follows that two additive functionals with the same associated measure have the same $p_{\alpha}$, hence the same $\alpha^{-1}\left(1-p_{\alpha}\right)=E \cdot\left[\mathrm{f}_{\alpha}\left(\mathrm{m}_{\partial D}\right)\right]$ $\left(<\alpha^{-1}<+\infty\right)$, and hence the same $f_{\alpha}$. But this means that the two functionals are the same up to time $m_{\partial D}$, and, to finish the proof, it is enough to make $D$ swell out to $R^{d}$. 


\section{CONSTRUCTION OF AN ADDITIVE FUNCTIONAL FROM ITS ASSOCIATED MEASURE.}

Given a smooth non-negative measure $e$, our task is to find an additive functional $\mathrm{f}$ with $e$ as its associated measure.

Consider, for this purpose, a non-negative measure $e$ on a bounded open figure $D$ with bounded potential $p=\int G d e$ and finite energy $\mathfrak{F}(e)=\int G d e d e$, let $p_{n}$ be the potential $\int G f_{n} d b$ of

$5.1 f_{n}=n\left(p-E \cdot\left[p\left(x\left(n^{-1}\right)\right), n^{-1}<m_{\partial D}\right]\right)$,

let

$5.2 \quad \mathrm{f}_{n}(t)=\int_{0}^{t \wedge \mathrm{m}^{2} D} f_{n}(x(s)) d s$,

and let us construct a functional $f$ associated with $e$ as a limit of $f_{n}$ with the aid of the following simple lemmas:

a) $p$ is a Brownian excessive function; esp. $0 \leq f_{n}$.

b) $E \cdot\left[p(x(t)), t<\mathrm{m}_{\partial D}\right] \downarrow 0$ inside $D$ as $t \uparrow+\infty$.

c) $p_{n}=n \int_{0}^{n^{-1}} E \cdot\left[p(x(t)), t<\mathfrak{m}_{\partial D}\right] d t$ increases to $p$ inside $D$ as $n \uparrow$ $+\infty$.

d) $\lim _{n \uparrow+\infty}\left(\mathfrak{F}\left(e-f_{n} d b\right)=0\right.$, where $\left(\mathfrak{F}\right.$ is the energy $\left(\mathfrak{F}(e) \equiv \int G d e d e\right.$.

e) $E \cdot\left[\mathfrak{f}_{n}(+\infty) \mid \boldsymbol{B}_{t \wedge \mathfrak{m}_{\partial D^{+}}}\right] \equiv \mathfrak{l}_{n}(t)$

$=p_{n}(x(t))+\mathfrak{f}_{n}(t) \quad \mathfrak{m}_{\partial D}>t \geq 0$

$=\mathrm{f}_{n}\left(\mathrm{~m}_{\partial D}\right) \quad \mathrm{m}_{\partial D} \leq t$,

i.e., $\mathfrak{I}_{n}$ is a martingate with respect to the fields $\boldsymbol{B}_{t \wedge m_{\partial D^{+}}}$; moreover, $\mathfrak{l}_{n}$ is continuous in $t$.

f) $P \cdot\left[\max _{t \geq 0}\left|\mathfrak{I}_{n}\left(t, w_{s}^{+}\right)-\mathfrak{l}_{m}\left(t, w_{s}^{+}\right)\right|>\varepsilon\right]$

$\leq$ constant $\times \varepsilon^{-2} s^{-d / 2} \sqrt{\mathfrak{F}\left(f_{n} d b-f_{m} d b\right)}$.

g) $P \cdot\left[\lim _{n \uparrow+\infty} f_{n}\left(t, w_{s}^{+}\right)=\mathfrak{f}\left(t, w_{s}^{+}\right), t \geq s>0\right]=1$.

where the limit is taken as $n \uparrow+\infty$ via suitable $n_{1}<n_{2}<$ etc., $\mathrm{f}(t)$ is continuous, $\mathrm{f}(0) \equiv \mathrm{f}(0+)=0$, and $\mathrm{f}(t)=\mathrm{f}(s)+\mathrm{f}\left(t-s, w_{s}^{+}\right)\left(\mathrm{m}_{\partial D} \geq t \geq s\right)$. h) $E \cdot\left[f\left(\mathrm{~m}_{\partial D}\right)\right]=p$.

Because $p$ is a potential, it is excessive; a) is obvious from this, $b$ ) is obvious from the bound 
$5.3 E \cdot\left[p(x(t)), t<m_{\partial D}\right] \leq\|p\|_{\infty} P \cdot\left(t<m_{\partial D}\right) \downarrow 0 \quad t \uparrow+\infty$,

and c) follows from $b)$ :

$5.4 \quad p_{n}=E \cdot\left[\int_{0}^{\mathfrak{n} t \partial D} f_{n}(x(s)) d s\right]$

$$
\begin{aligned}
= & n \int_{0}^{+\infty} d s E \cdot\left[p(x(s))-E_{x(s)}\left[p\left(x\left(n^{-1}\right)\right), n^{-1}<m_{\partial D}\right], s<\mathfrak{m}_{\partial D}\right] \\
= & n \int_{0}^{+\infty} d s\left[E \cdot\left[p(x(s)), s<m_{\partial D}\right]-E \cdot\left[p\left(x\left(s+n^{-1}\right)\right), s+n^{-1}\right.\right. \\
& \left.\left.<m_{\partial D}\right]\right] \\
= & n \int_{0}^{n^{-1}} E \cdot\left[p(x(s)), s<m_{\partial D}\right] d s \uparrow p \quad n \uparrow+\infty .
\end{aligned}
$$

An application of c) establishes

$5.5 \quad \mathfrak{F}(e)=\int p d e \geq \int p_{n} d e=\int p f_{n} d b \geq \mathfrak{F}\left(f_{n} d b\right) \uparrow(\mathfrak{F}(e) \quad n \uparrow+\infty$,

and this implies d):

5. $6 \lim _{n \uparrow+\infty}\left(5\left(e-f_{n} d b\right)=\lim _{n+\uparrow}\left[\mathfrak{G}(e)-2 \int p_{n} d e+\mathfrak{S}\left(f_{n} d b\right)\right]=0\right.$.

Because $t \bigwedge \mathfrak{m}_{\partial D}$ is a Markov time,

$5.7 E \cdot\left[\mathfrak{f}_{n}\left(\mathfrak{M}_{\partial D}\right) \mid B_{t \wedge \mathfrak{m}_{\partial D^{+}}}\right]=E \cdot\left[\int_{t \wedge \mathfrak{m} \partial D}^{\mathfrak{m}_{\partial \supset}} f_{n}(x(s)) d s \mid B_{t \wedge \mathfrak{m}_{\partial D^{+}}}\right]+\mathfrak{f}_{n}(t)$

$$
=E_{x\left(t \wedge \mathfrak{m}_{\partial D}\right)}\left[\int_{0}^{\mathfrak{m}_{\partial D}} f_{n} d s\right]+\mathfrak{f}_{n}(t)=p_{n}(x(t))+\mathfrak{f}_{n}(t) \equiv \mathfrak{l}_{n}(t) \quad t<\mathfrak{m}_{\partial D},
$$

i.e., $\mathfrak{l}_{n}$ is martingale, and since $p_{n}$ is continuous and tends to 0 along the Brownian path $\left(t \uparrow m_{\partial D}\right), \mathfrak{l}_{n}$ is continuous. e) is now established, and f) follows from Doob's submartingale extension of Kolmogorov's inequality [4], the Schwarz inequality

$5.8 \quad\left(\int G d e_{1} d e_{2}\right)^{2} \leq \mathfrak{F}\left(e_{1}\right) \mathfrak{F}\left(e_{2}\right)$

(see H. Cartan [2]), and the resulting

5. 9

$$
\begin{aligned}
& E \cdot\left[\left|\mathfrak{l}_{n}\left(+\infty, w_{s}^{+}\right)-\mathfrak{l}_{m}\left(+\infty, w_{s}^{+}\right)\right|^{2}, s<\mathrm{m}_{\partial D}\right] \\
& \leq E \cdot\left[E_{x(s)}\left[\left|\mathfrak{f}_{n}\left(\mathfrak{m}_{\partial D}\right)-\mathfrak{f}_{m}\left(\mathfrak{m}_{\partial D}\right)\right|^{2}\right], s<\mathfrak{m}_{\partial D}\right] \\
& \leq E \cdot\left[\int G(x(s), b)\left(f_{n}-f_{m}\right)\left(p_{n}-p_{m}\right) d b, s<\mathfrak{m}_{\partial D}\right]
\end{aligned}
$$




$$
\begin{aligned}
& =\iint G(a, b) e_{n m}(d a) E \cdot\left[G(x(s), b), s<m_{\partial D}\right] e_{n m}(d b) \\
& \leq \mathfrak{F}\left(e_{n m}\right)^{1 / 2} \mathfrak{F}\left(E \cdot\left[G(x(s), b), s<\mathrm{m}_{\partial D}\right] d e_{n m}\right)^{1 / 2} \\
& \leq \mathfrak{F}\left(e_{n m}\right)^{1 / 2} \text { constant } \times s^{-d / 2} \mathfrak{F}\left(e_{n m}\right)^{1 / 2} .
\end{aligned}
$$

Choose $n_{1}<n_{2}<$ etc. so as to make $P \cdot\left[\max _{t \geq 0}\left|\mathfrak{l}_{n}\left(t, w_{s}^{+}\right)-\mathfrak{l}_{s}(t)\right| \downarrow 0\right.$, $s>0]=1$, where $\mathfrak{l}_{s}$ is a continuous function of $t$. Because $p=\lim _{n \uparrow \infty+} p_{n}$ is continuous and tends to 0 along the Brownian path $\left(t \uparrow \mathrm{m}_{\partial D}\right)$, it follows that $\mathrm{f}_{s}(t)=\lim _{n \uparrow+\infty} \mathrm{f}_{n}\left(t, w_{s}^{+}\right)$is continuous $(t \geq 0)$ and additive $\left(t \leq \mathfrak{m}_{\partial D}\left(w_{s}^{+}\right)\right)$; moreover

5.10

$$
E \cdot\left[f_{n}(+\infty)^{2}\right]=2 \int G p_{n} f_{n} d b \leq 2\|p\|_{\infty}^{2}<+\infty
$$

implies

5.11

$$
\begin{aligned}
& E \cdot\left[f_{s}(t-s)\right]=\lim _{n \uparrow+\infty} E \cdot\left[f_{n}\left(+\infty, w_{s}^{+}\right)-f_{n}\left(+\infty, w_{t}^{+}\right)\right] \\
& =\lim _{n \uparrow+\infty}\left[E \cdot\left(p_{n}(x(s)), s<m_{\partial D}\right)-E \cdot\left(p_{n}(x(t)), t<m_{\partial D}\right)\right] \\
& =E \cdot\left(p(x(s)), s<m_{\partial D}\right)-E \cdot\left(p(x(t)), t<m_{\partial D}\right),
\end{aligned}
$$

and, to finish the proof of $\mathrm{g}$ ) and $\mathrm{h}$ ), it is enough to define

$$
\text { 5. } 12 \quad f(t)=\lim _{s \downarrow 0} f_{s}(t)
$$

and to make $s \downarrow 0$ and $t \uparrow+\infty$ in 5.11.

Now take a smooth measure $e$, choose $B_{n} \uparrow D$ as needed for 1.8 with the additional property that $e \mid B_{n}$ has finite energy $\mathfrak{F}\left(e \mid B_{n}\right)=\int_{B_{n} \times B_{n}} G d e d e^{6}$, let $f_{n}$ be the additive functional associated with $e \mid B_{n}$ as in 5.12 above, and note that

$$
5.13 \quad E \cdot\left[\int_{0}^{\mathfrak{m}_{\partial D}} f d f_{n}\right]=\int_{B_{n}} G f d e \quad f \geq 0
$$

as in e) of section 3. It follows that if $f$ is the indicator function

$6 \Leftrightarrow\left(e \mid B_{n}\right)<+\infty$ is achieved as follows : take $\dot{D} \supset D$ with $\partial \dot{D}$ at a positive distance from $\partial D$, choose $\dot{B}_{n} \uparrow \dot{D}$ as needed for 1.8 , and let $B_{n}=\dot{B}_{n} \cap D$; then $\&\left(e \mid B_{n}\right) \leq$ $\int_{B_{n}} d e \int_{B_{n}} \dot{G} d e \leq n e\left(B_{n}\right) \leq n\left(\inf _{D \times D} \dot{G}\right)^{-1} \sup _{D} \int_{B_{n}} \dot{G} d e \leq n^{2}\left(\inf _{D \times D} \dot{G}\right)^{-1}<+\infty$. 
of $B_{m}$, then the functionals $\int_{0}^{t} f d \mathrm{f}_{n}(n>m)$ and $\int_{0}^{t} f d \mathrm{f}_{m}$ have the same (bounded) mean :

$5.14 E \cdot\left[\int_{0}^{\mathfrak{m} \partial D} f d f_{n}\right]=E \cdot\left[\int_{0}^{\mathfrak{m} \partial D} f d f_{m}\right]=\int_{B_{m}} G d e$

and are therefore identical up to time $m_{\partial D}$ according to the first uniqueness lemma for additive functionals of section 4 . But this means that $\mathrm{f}_{n}=\mathrm{f}_{m}$ up to the exit time from $B_{m}$, and since this exit time $=\mathfrak{m}_{\partial D}$ for large $m$, it is legitimate to define a functional $f$ for $t \leq \mathfrak{m}_{\partial D}$ by means of

$5.15 \mathrm{f}(t)=\mathrm{f}_{n}(t) \quad t \leq \inf \left(t: x(t) \notin B_{n}\right), n \geq 1$.

Introducing $p_{\alpha}=E \cdot\left[e^{-\alpha f\left(\mathfrak{m}_{\partial D}\right)}\right]$ and using the method of 3.5, 5.13 implies

5.16

$$
\begin{aligned}
& 1-p_{\infty}=\lim _{n \uparrow+\infty} \alpha E \cdot\left[\int_{0}^{\mathrm{n} \partial \partial} e^{\left.-\alpha\left[\mathfrak{f}\left(\operatorname{m}_{\partial D}\right)-\mathfrak{f}(t)\right]_{\mathfrak{f}_{n}}(d t)\right]}\right. \\
& =\lim _{n \uparrow+\infty} \alpha E \cdot\left[\int_{0}^{\mathrm{n} \partial D} p_{a} d \mathfrak{f}_{n}\right]=\lim _{n \uparrow+\infty} \alpha \int_{B_{n}} G p_{\alpha} d e=\alpha \int G p_{\alpha} d e ;
\end{aligned}
$$

in brief, $e$ is the measure associated with $f$ as in 3.2.

Because two additive functionals with same associated measure are the same, it follows that if $e$ is smooth and if $D_{1} \subset D_{2}<$ etc. swell out to $R^{d}$, then the functionals $f_{n}$ associated with $e_{n}=e \mid D_{n}$ as in 5.15 above determine an additive functional

$5.17 \mathrm{f}(t)=\mathrm{f}_{n}(t) \quad t<\mathrm{m}_{\partial D_{n}}, n \geq 1$

depending upon $e$ alone, for which 3.2 holds for each $D$.

$f$ is additive for almost all Brownian paths, but of course it can be modified on a negligeable class of paths so as to be Borel in the pair $(t, w)$ and to satisfy $1.1,1.2,1.3,1.4$, as identities; with this modification, $f$ is the additive functional associated with $e$.

\section{DIFFUSIONS WITH BROWNIAN HITTING PROBABILITIES.}

To avoid confusion, let $w, x, \mathrm{~m}, B, \boldsymbol{B}, \boldsymbol{B}_{\mathfrak{m}^{+}}$, etc. be used to describe the Brownian motion, introduce the same paths, times, events, and fields with the new names $\dot{w}, \dot{x}, \dot{\mathrm{n}}, \dot{B}, \dot{\boldsymbol{B}}, \dot{\boldsymbol{B}}_{\mathrm{in}^{+}}$, etc., and take a new motion $\dot{\boldsymbol{D}}$ with probabilities $\dot{P}_{a}(\dot{B})$. 
$\dot{\boldsymbol{D}}$ is said to be a diffusion if it starts afresh at each Markov time nil:

6. $1 \quad \dot{P}_{a}\left[\dot{w}_{\mathfrak{i l}}^{+} \in \dot{B} \mid \dot{B}_{\mathfrak{i l}^{+}}\right]=\dot{P}_{b}(\dot{B}) \quad a \in E^{d}, \dot{B} \in \dot{B}, b \equiv \dot{x}(\dot{i}) ;$

it is said to have Brownian hitting probabilities if, for each bounded open $D$,

6. $2 \quad \dot{P}_{a}\left[\dot{x}\left(\dot{m}_{\partial D}\right) \in d b\right]=P_{a}\left[x\left(\mathfrak{m}_{\partial D}\right) \in d b\right]=h_{\partial D}(a, d b)$

$$
a \in D, d b<\partial D \text {. }
$$

Given such a diffusion with Brownian hitting probabilities

6. 3a $\dot{p}_{\omega} \equiv \dot{E} \cdot\left[e^{-\alpha \dot{n} \partial \nu}\right]^{7}$

solves

6. 3b $1-\dot{p}_{a}=\alpha \int G \dot{p}_{a} d e$,

where $e \geq 0$ is independent of $\alpha$ and of $D . \quad e$ is the so-called speed measure of the diffusion; the speed measure of the Brownian motion is the Lebesgue measure $d b$. Because of $6.2, \dot{P} \cdot\left[\operatorname{li}_{\partial D}<\right.$ $+\infty]=1$ and $\dot{p}_{\infty}>0$, which implies that $e$ is smooth, i.e., that it is the associated measure of some additive functional $f$ of the Brownian path; moreover, $e$ is positive on the neighborhoods of H. Cartan's fine topology, and this implies that its associated additive functional satisfies

6. $4 P \cdot[\mathfrak{f}(s)<\mathfrak{f}(i), 0 \leq s<t]=1$.

Introducing the inverse function $\mathrm{f}^{-1}$ of $\mathrm{f}$, it turns out that

$6.5 \dot{P} \cdot(\dot{B})=P \cdot\left[x\left(\mathfrak{f}^{-1}\right) \in \dot{B}\right] \quad \dot{B} \in \dot{B}$;

in brief, $\dot{\boldsymbol{D}}$ is identical in law to the Brownian motion run with the stochastic clock $\mathrm{f}^{-1}$; moreover, each smooth measure $e$, positive on fine neighborhoods, is the speed measure of a diffusion with Brownian hits.

The proofs are carried out in the next few sections.

${ }^{7} \dot{E} .(f)=\int f d \dot{P}$. 


\section{SPEED MEASURES}

Beginning with the speed measure $e$ of $6.3 \mathrm{~b}$, if a $a \in R^{d}$ and if $\dot{n i}_{\varepsilon}=\min (t:|\dot{x}(t)-a| \geq \varepsilon)$, then $\dot{n i}_{0+}=\lim _{\varepsilon \downarrow 0} \dot{u}_{\varepsilon}$ satisfies

7.1

$$
\dot{E}_{a}\left(e^{-\dot{\mathfrak{m}}_{0+}}\right)=\dot{E}_{a}\left[e^{-\dot{\mathrm{m}}-\dot{\mathrm{m}}\left(w_{\dot{\mathrm{m}}}^{+}\right)}\right]=\dot{E}_{a}\left(e^{-\dot{\mathrm{m}}_{0+}}\right)^{2}, \quad \dot{\mathrm{n}}=\mathfrak{n}_{0+}
$$

and since $\dot{E}_{a}\left(e^{-\dot{m}_{0+}}\right)=0$ implies $\dot{P}_{a}\left[\dot{n}_{0+}=+\infty\right]=1$, violating the fact that the dot motion has Brownian hitting probabilities, it follows that $\dot{P}_{a}\left[\dot{1}_{0+}=0\right]=1$.

Because

$\left.7.2 \quad 1-\dot{p}_{\alpha}=\alpha \dot{E} \cdot\left[\int_{0}^{\dot{u} \partial D} e^{-\alpha(\dot{m} \partial D D}-t\right) d t\right]=\alpha \dot{E} \cdot\left[\int_{0}^{\dot{i} \partial D} \dot{p}_{\alpha}(x(t)) d t\right]$, $1-\dot{p}_{a}$ satisfies

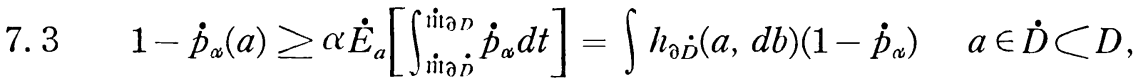
and, using 7.3 and $\dot{P}_{a}\left[{ }^{-} i_{0+}=0\right]=1$ to establish

7. $4 \mathrm{a} \quad \int h_{\partial \dot{D}}(a, d b)\left(1-\dot{p}_{a}\right) \downarrow 0 \quad \dot{D} \uparrow D$

7. $4 \mathrm{~b} \int h_{|b-a|=\varepsilon}(a, d b)\left(1-\dot{p}_{a}\right) \uparrow 1-\dot{p}_{a} \quad \varepsilon \downarrow 0$,

it appears that $1-\dot{p}_{\alpha}$ is the potential $\alpha \int G d e_{\alpha}$ of a non-negative charge distribution $\alpha e_{\omega}$

It remains to verify that $\dot{p}_{\alpha}^{-1} d e_{\alpha} \equiv d e$ is independent of $\alpha$ and of $D$, which is done with the aid of the additive functional $\dot{\hat{f}}_{\alpha}(t)$ $=\int_{0}^{t} \dot{p}_{a}(\dot{x}(s)) d s\left(t<\dot{n}_{\partial D}\right)$ and the method of section 3 ; in outline,

$7.5 \quad 1-\dot{p}_{a}=\alpha \dot{E}_{\alpha}\left[\dot{\mathfrak{f}}_{a}\left(\dot{i}_{\partial D}\right)\right]=\alpha \int G d e_{c}$

implies

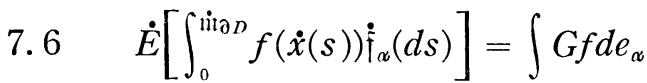

as in e) of section 3 , and 3.9 follows as before, etc..

8. TWO DIFFUSIONS WITH BROWNIAN HITS AND THE SAME SPEED MEASURE ARE THE SAME.

Consider a pair of diffusions with Brownian hitting probabilities and the same speed measure. 
Because $6.3 \mathrm{~b}=3.2$ has unique solutions as noted in section 4 , both diffusions have the same $\dot{p}_{\alpha}=\dot{E}$. $\left[e^{-\alpha \dot{r i t} \nu}\right]$ and hence the same 8. $1 \quad \dot{G}_{0^{+}}: f \rightarrow \dot{E} \cdot\left[\int_{0}^{\dot{m} \partial D} f(\dot{x}) \dot{\hat{f}}_{\varepsilon}(d t)\right]=\int G f \dot{p}_{\varepsilon} d e$

$$
\dot{\mathrm{f}}_{\varepsilon}(t) \equiv \int_{0}^{t} \dot{p}_{\varepsilon}(\dot{x}(s)) d s \quad t \leq \dot{\mathrm{m}}_{\partial D} .
$$

Choose $\varepsilon>0$ and introduce the Green operators

8. $2 \quad \dot{G}_{\alpha}: f \rightarrow \dot{E} \cdot\left[\int_{0}^{\dot{\mathrm{m}}_{\partial} \partial} e^{-\alpha \dot{\mathrm{f}}_{\varepsilon}(t)} f(\dot{x}) \dot{\mathfrak{f}}_{\varepsilon}(d t)\right] \quad \alpha>0$;

then

8. $3 \alpha \dot{G}_{0+} \dot{G}_{\alpha} f=\alpha \dot{E} \cdot\left[\int_{0}^{\dot{m} \partial \partial D} \dot{\mathfrak{f}}_{\varepsilon}(d t)\left(\dot{G}_{a} f\right)(\dot{x})\right]$

$$
\begin{aligned}
& =\alpha \dot{E} \cdot\left[\int_{0}^{\dot{i} \partial D} \dot{f}_{\varepsilon}(d t) \int_{t}^{\dot{r i} \partial D} e^{-\alpha\left[\dot{f}_{\varepsilon}(s)-\dot{f}_{\varepsilon}(t)\right]} f(\dot{x}(s)) \dot{f}_{\varepsilon}(d s)\right] \\
& =\alpha \dot{E} \cdot\left[\int_{0}^{\dot{m} \partial D} e^{-\alpha \dot{f}_{\varepsilon}(s)} f(\dot{x}) \dot{f}_{\varepsilon}(d s) \int_{0}^{s} e^{\alpha \dot{\mathfrak{f}}_{\varepsilon}} d \dot{\mathfrak{f}}_{\varepsilon}\right] \\
& =\dot{E} \cdot\left[{ }_{0}^{\dot{r} \jmath_{\partial} D}\left(1-e^{-\alpha \dot{f}_{\varepsilon}}\right) f d \dot{\mathfrak{f}}_{\varepsilon}\right]=\dot{G}_{0+} f-\dot{G}_{\alpha} f \text {, }
\end{aligned}
$$

i.e.,

8. $4 \quad \dot{G}_{a}=\dot{G}_{0+}-\alpha \dot{G}_{0+} \dot{G}_{\alpha}$,

and, using the bound

8. $5 \quad \dot{G}_{0+} 1=\int G \dot{p}_{\mathrm{\varepsilon}} d e=\varepsilon^{-1}\left(1-\dot{p}_{\mathrm{q}}\right) \leq \varepsilon^{-1}<+\infty$,

the obvious iteration of 8.4 implies

8. $6 \quad \dot{G}_{a}=\sum_{n \geq 0}(-)^{n} \alpha^{n} \dot{G}_{0+}^{n+1} \quad \alpha<\varepsilon$.

Because $\dot{G}_{\alpha}$ is a Laplace transform in its dependence on $\alpha$, 8.6 implies that both diffusions have the same Green operators $(\alpha \geq 0)$ and hence the same

8. $7 \quad \dot{E} \cdot\left[\int_{0}^{\dot{n}_{\infty}} e^{-\alpha t} f(\dot{x}) d t\right]=\lim _{D \uparrow R^{d}} \lim _{\varepsilon \downarrow 0} \dot{G}_{\alpha} f$.

But this implies that both diffusions have the same 8. $8 \quad \dot{P}_{a}\left[\dot{x}(t) \in d b, t<\sim_{\infty}\right] \quad(t, a, b) \in(0,+\infty) \times R^{2 d}$ and hence are the same in all respects as stated in the section title. 


\section{SPEED MEASURES ARE POSITIVE ON FINE NEIGHBORHOODS.}

Given a point $a$ of bounded open $D$ and a (Brownian) excessive function $p$ on $D$, the set of points $b \in D$ at which $|p(b)-p(a)|<n^{-1}$ is said to be a fine neighborhood of $a$; the corresponding topology is called the fine topology of H. Cartan [2]. E. B. Dynkin [7] has pointed out that a point $a$ is in the fine interior of $B \subset R^{d}$ if and only if almost all Brownian paths starting at $a$ remain in $B$ for some positive time. Because an excessive function in 1-dimension is concave and hence continuous, the 1-dimensional fine topology is the same as the usual one; in higher dimensions it is different.

Given a diffusion with Brownian hitting probabilities, its speed measure e has to be positive on fine neighborhoods; for the proof, it is enough to verify that if $Z \subset R^{d}$ is bounded and Borel and if $e(Z)=0$, then $Z$ has no fine interior.

Choose an open ball $D$ and a decreasing series of open figures $D_{n}$ so as to have

9.1a $\quad D \supset D_{1} \supset D_{2} \supset$ etc. $\supset Z$

$9.1 \mathrm{~b} \int_{D_{n}} G_{D} \dot{p}_{1} d e \downarrow 0 \quad n \uparrow+\infty, \dot{p}_{1}=\dot{E} \cdot\left[e^{-\dot{m}_{\partial} D}\right]$, and let

$9.2 \quad \dot{\mathfrak{f}}_{1}(t)=\int_{0}^{t} \dot{p}_{1}(\dot{x}(s)) d s \quad t \leq \mathfrak{\eta}_{\partial D}$.

Because of

9. 3a $\quad 1-\dot{E}_{a}\left[e^{-\dot{\mathfrak{f}}_{1}\left(\partial \mathrm{ar} D_{n}\right)}\right]=\int_{D_{n}} G_{D_{n}} \dot{p}_{1} d e \leq \int_{D_{n}} G_{D} \dot{p}_{1} d e \downarrow 0$

9. 3b $\quad \dot{p}_{1}>0$, $n \uparrow+\infty, a \in Z$

it is apparent that

9. $4 \mathrm{a} \quad 1=\dot{P}_{a}\left[\operatorname{ri}_{\partial D_{n} \downarrow} \downarrow 0, n \uparrow+\infty\right]$

$=\dot{P}_{a}\left[\lim _{n \uparrow+\infty} \dot{x}\left(\dot{n}_{\partial D n}\right)=a\right]$

$=\lim _{n \uparrow+\infty} \int h_{\partial D_{n}}(a, d b) e^{-|b-a|} \quad a \in Z$.

But this final expression depends upon the (Brownian) hitting 
probabilities $h_{\partial D}$ alone; thus,

$9.4 \mathrm{~b}$

$$
P_{a}\left[\lim _{n \uparrow+\infty} x\left(\mathrm{~m}_{\partial D}\right)=a\right]=1 \quad a \in Z
$$

for the Brownian motion also, and since almost no Brownian path meets its starting point at a positive time, it follows that

9.5 $\quad P_{a}\left[\mathrm{~m}_{\partial D_{n}} \downarrow 0, n \uparrow+\infty\right]=1 \quad a \in Z$;

an application of Dynkin's description of fine neighborhoods completes the proof.

\section{SPEED MEASURES GIVE RISE TO INCREASING ADDITIVE FUNCTIONALS.}

Because the speed measure of a diffusion with Brownian hits is smooth, it has associated with it an additive functional $f$ of the Brownian path; moreover, $e$ is positive on fine neighborhoods, and this is reflected in the fact that $f$ is increasing as in 6.4: $\mathrm{f}(s)<\mathrm{f}(t)(s<t)$.

It will be enough to verify that the set $A$ of points $a$ at which

10.1 $P_{a}(\mathrm{~m}>0)=P_{a}[\mathrm{f}(t)=0$ for some $t>0]=1$

$$
\mathrm{m}=\inf (t: f(t)>0)
$$

is vaccuous; note that $A$ is Borel and that $P .(m>0)=0$ or 1 according to Blumenthal's 01 law.

Because $A$ is either void or fine open and $e$ is positive on fine neighborhoods, it is enough to show that $e(A)=0$, and, for this, it is enough to show that for each bounded open $D$, the points of $D$ at which $P \cdot\left[\mathrm{f}\left(\mathrm{m}_{\partial D}\right)>0\right]<1$ have $e$-mass 0 . But this is immediate on letting $\alpha \uparrow+\infty$ in

$10.2 \alpha^{-1}\left(1-p_{a}\right)=\int G p_{\alpha} d e \quad p_{a}=E \cdot\left[e^{-\alpha \uparrow\left(\mathrm{m}_{\partial} p\right)}\right]$.

11. WHEN IS $f(+\infty)=+\infty$ ?

In making up the stochastic clock $f^{-1}$ for use in 6.5 , two cases arise according as $f(+\infty)=+\infty$ or not, and it is desirable to have a test for this.

Because $p \equiv P \cdot[\mathrm{f}(+\infty)<+\infty]$ satisfies 
$11.1 p(a)=P_{a}\left[\mathrm{f}\left(\mathrm{m}_{\partial D}\right)<+\infty, \mathrm{f}\left(+\infty, w_{\mathrm{m}_{\partial D}}^{+}\right)<+\infty\right]$

$$
=E_{a}\left[p\left(x\left(\mathfrak{m}_{\partial D}\right)\right)\right]=\int h_{\partial D}(a, d b) p(b) \quad a \in D
$$

for bounded open $D$, it is harmonic and since $p \geq 0$, it must be constant; moreover, letting first $t$ and then $n \uparrow+\infty$ in

$$
P \cdot[\mathrm{f}(+\infty)<n] \leq E \cdot[\mathrm{f}(t)<n, p(x(t))] \leq P \cdot[\mathrm{f}(t)<n] p,
$$

it appears that $p \leq p^{2}$ and hence that $p=0$ or 1 .

Our test states that $p=1$ if and only if one of the following conditions is met:

a) $d \geq 3$ and $1-p_{1}=\int G p_{1}$ de admits a solution $0<p_{1} \leq 1$ on the whole of $R^{d}$, where $G$ is the function of $R^{d}$.

b) $\quad\left(S p_{1}=p_{1}\right.$ admits a solution $0<p_{1} \leq 1$ on the whole of $R^{d}$, where (S $p_{1}$ is the negative of the Radon-Nikodym derivative of the Riesz measure of $p_{1}$ with respect to $e$ (see section 13 for the meaning of (S). c) $\quad d \geq 3$ and $R^{d}=A \cup B$, where $A$ is thin at $\infty$ in the sense that $P .[x(t) \in A$ for some $t>n] \downarrow 0(n \uparrow \infty)$, and $\int_{B} G d e<+\infty .^{8}$

Beginning with $p=1, p_{1} \equiv E \cdot\left[e^{-f(+\infty)}\right]$ is positive and $\leq 1$, and, since $G_{D} \uparrow G$ as $D \uparrow R^{d}$,

$$
\begin{gathered}
11.31-p_{1}=\lim _{D \uparrow R^{d}} E \cdot\left[e^{-\mathrm{f}\left(+\infty, w_{\mathfrak{m} D}^{+}\right)}\right]-p_{1} \\
=\lim _{D \uparrow R^{d}} \int G_{D} p_{1} d e=\int G p_{1} d e .
\end{gathered}
$$

Because $G \equiv+\infty$ in case $d=2$, it follows that $p=1$ implies a), that a) implies b) is clear, that c) implies $p=1$ is evident from

$$
11.4 E \cdot\left[\int_{0}^{+\infty} f d \uparrow\right]=\int_{B} G d e<+\infty \text {, }
$$

where $f$ is the indicator function of $B$, and now it remains to verify that b) implies c).

But, if $0<p_{1} \leq 1$ is a solution of $\left(\beta_{1}=p_{1}\right.$ and if $h_{1}$ is its

$8 A \subset R^{d}(d \geq 3)$ is thin at $\infty$ if and only if (Wiener's test) $\sum_{n \geq 1} 2^{-n(a-2)} C\left(A_{n}\right)<$ $+\infty$, where $A_{n}$ is the meet of $A$ with the spherical $2^{n-1}|b|<2^{n}$ and $C$ is the $d$ dimensional Newtonian capacity; for the proof in the case of the $d$-dimensional random walk, see K. Itô and H. P. McKean, Jr. [12]. 
harmonic part $E \cdot\left[p_{1}\left(x\left(\mathfrak{m}_{\partial D}\right)\right)\right]$ inside $D$, then $h_{1}-p_{1}=\int G_{D} p_{1} d e$ inside $D$, and, as $D \uparrow R^{d}, h_{1}$ decreases to a non-negative (and hence constant) harmonic function $p_{1}(\infty)$ such that $p_{1}(\infty)-p_{1}=\int G p_{1} d e . \quad R^{d}$ is now split into $A=\left(p_{1}<\frac{1}{2} p_{1}(\infty)\right)$ and $B=\left(p_{1} \geq \frac{1}{2} p_{1}(\infty)\right)$ and the fact that $p_{1}(\infty)-p_{1}$ excessive is used to ensure that $p_{1}$ has a limit along the Brownian path as $t \uparrow+\infty$, permitting us to conclude from

11. $4 \mathrm{a} \quad p_{1} \leq p_{1}(\infty)$

11. 4b $p_{1}(\infty)=\lim _{D \uparrow R^{d}} E \cdot\left[p_{1}\left(x\left(\mathrm{~m}_{\partial D}\right)\right)\right]=E \cdot\left[\lim _{t \uparrow+\infty} p_{1}(x(t))\right]$

that $A$ is thin at $\infty$. Because $0<p_{1}(\infty)$,

11.5

$$
\int_{B} G d e \leq 2 p_{1}(\infty)^{-1} \int G p_{1} d e<+\infty,
$$

and this completes the verification of $\mathrm{c}$ ).

\section{PERFORMING THE TIME SUBSTITUTION.}

Coming to the actual time substitution $t \rightarrow f^{-1}$ which is supposed to send the Brownian motion into the diffusion $\dot{\boldsymbol{D}}$, let $f$ be modified on a negligeable class of Brownian paths so as to have

$12.10=\mathrm{f}(0) \leq \mathrm{f}(t \pm)=\mathrm{f}(t)<+\infty$

12.2 $\mathrm{f}(t)=\mathrm{f}(s)+\mathrm{f}\left(t-s, w_{s}^{+}\right) \quad t \geq s$

12. $3 \mathrm{f}(t)>\mathrm{f}(s) \quad t>s$

as identities, let $x^{-1}$ denote the sample path

12. $4 w^{-1}: t \rightarrow x^{-1}(t) \equiv x^{-1}\left(t, w^{-1}\right) \equiv x\left[f^{-1}(t, w), w\right]$,

note that this path is continuous even if $f(+\infty)<+\infty(d \geq 3)$, and let us check that the motion $\dot{\boldsymbol{D}}$ with sample paths

12. $5 \mathrm{a} \quad \dot{w}: t \rightarrow \dot{x}(t)$

\section{and probabilities}

12.5b $\quad \dot{P}_{a}(\dot{B}) \equiv P_{a}\left(w^{-1} \in \dot{B}\right)$

is the diffusion with Brownian hitting probabilities and speed measure $e$.

Beginning with the proof of the diffusive character 6.1 of this 
motion, the problem is to check that if $i$ is a Markov time and if $\dot{B} \in \dot{\boldsymbol{B}}$, then

12. $6 \quad \dot{P}_{a}\left[\dot{w}_{\dot{\mathfrak{m}}}^{+} \in \dot{B} \mid \dot{B}_{\dot{\mathfrak{m}}^{+}}\right]=\dot{P}_{b}(\dot{B}) \quad b \equiv \dot{x}(\mathfrak{i n})$.

Given such a Markov time in,

12. $7 \quad \mathfrak{m}(w) \equiv \mathfrak{f}^{-1}\left(\mathfrak{m}\left(w^{-1}\right), w\right)$

is a Markov time for the Brownian path ; indeed, using Galmarino's test, if

12. $8 \mathrm{a} \quad \mathrm{m}(u)<t$

12. $8 \mathrm{~b} \quad x(s, u)=x(s, v) \quad s \leq t$,

then

12.9a $\mathrm{f}(s, u)=\mathrm{f}(s, v) \quad s \leq t$

12.9b $\quad \mathrm{f}^{-1}(s, u)=\mathrm{f}^{-1}(s, v) \leq t \quad s \leq \dot{t}=\mathrm{f}(t, u)=\mathrm{f}(t, v)$,

and it follows that

12. 10a $\operatorname{m}\left(u^{-1}\right)=\mathrm{f}(\mathrm{m}(u), u)<\mathrm{f}(t, u)=\dot{t}$

12.10b $\quad x^{-1}\left(s, u^{-1}\right)=x\left[f^{-1}(s, u), u\right]=x\left[f^{-1}(s, v), v\right]$ $=x^{-1}\left(s, v^{-1}\right) \quad s \leq \dot{t}$.

Because nit was a Markov time for the dot motion, 12.10 implies

12. 11a $\mathfrak{m}\left(u^{-1}\right)=\mathfrak{m}\left(v^{-1}\right)<\dot{t}$,

and an application of $12.9 \mathrm{~b}$ implies

12.11b $\operatorname{m}(u)=\mathfrak{f}^{-1}\left(\mathfrak{m}\left(u^{-1}\right), u\right)=\mathfrak{f}^{-1}\left(\mathrm{~m}\left(v^{-1}\right), v\right)=\mathrm{m}(v) ;$

in brief, 12.8 implies, $12.11 \mathrm{~b}$, as needed to conclude by Galmarino's test that $\mathrm{m}$ is a Markov time.

Given $\dot{A} \in \dot{\boldsymbol{B}}_{\mathrm{it}^{+}}$, if $A=\left(w: w^{-1} \in \dot{A}\right)$ and if

12. 12a $\mathfrak{m}(u)<t$

12.12b $x(s, u)=x(s, v) \quad s \leq t$

12. 12c $u \in A$, then, using 12.10 ,

12.13a $\operatorname{nin}\left(u^{-1}\right)<\dot{t}$

12.13b $\quad x^{-1}\left(s, u^{-1}\right)=x^{-1}\left(x, v^{-1}\right) \quad s \leq \dot{t}$

12.13c $u^{-1} \in A$, 
and it follows that $v^{-1} \in \dot{A}$, or, what is the same, that $v \in A$; thus, by Galmarino's test, $A \in \boldsymbol{B}_{\mathrm{m}+}$, and now it appears that

12.14 $\dot{P}_{a}\left[\dot{A}, \dot{w}_{\mathrm{nit}}^{+} \in \dot{B}\right]$

$$
\begin{aligned}
& =P_{a}\left[w^{-1} \in \dot{A},\left(w_{\mathfrak{l n}}^{+}\right)^{-1} \in \dot{B}\right] \\
& =P_{a}\left[w \in A,\left(w_{\mathfrak{m}}^{+}\right)^{-1} \in \dot{B}\right] \\
& =E_{a}\left[A, P_{b}\left(w^{-1} \in \dot{B}\right)\right] \quad b \equiv x(\mathfrak{m})=x^{-1}\left(\mathfrak{i n}\left(w^{-1}\right), w^{-1}\right) \\
& =E_{a}\left[w^{-1} \in \dot{A}, \dot{P}_{b}(\dot{B})\right] \\
& =\dot{E}_{a}\left[\dot{A}, \dot{P}_{b}(\dot{B})\right] \quad b=\dot{x}(\mathfrak{i n}),
\end{aligned}
$$

completing the proof of 12.6 .

$\dot{\boldsymbol{D}}$ is now identified as a diffusion; that it has Brownian hitting probabilities is clear, and to complete the discussion, it suffices to verify that it has e as its speed measure. But this is clear because 12. $15 \quad \mathrm{~m}_{\partial D}^{-1} \equiv \min \left(t: x^{-1}(t) \in \partial D\right)=\mathrm{f}\left(\mathrm{m}_{\partial D}\right)$, and $f$ has $e$ as its associated measure.

\section{GENERATORS.}

Given a diffusion with Brownian hitting probabilities, the Green operators

13. 1

$$
\dot{G}_{\alpha}: f \rightarrow \dot{E} \cdot\left[\int_{0}^{+\infty} e^{-\alpha t} f(\dot{x}) d t\right] \quad \alpha>0
$$

map into itself the space $\dot{C}\left(E^{d}\right)$ of real, bounded, fine-continuous functions having ordinary limits at $\infty$ in case $d \geq 3$; in fact, if $d \geq 3$, then $\dot{P} \cdot\left[\min _{t \geq 0}|\dot{x}(t)| \geq n\right]=P \cdot\left[\min _{t \geq 0}|x(t)| \geq n\right]$ tends to 1 at $\infty$, so that $\dot{G}_{\alpha} f$ tends to $\alpha^{-1} f(\infty)$, and, if $a \in R^{d}(d \geq 2)$ and if the ball $D \ni a$ is so small that $1-\dot{p}_{a}(a)=1-\dot{E} \cdot\left[e^{-\alpha \mathrm{ni} \partial p}\right]<n^{-1}$, then, inside the fine neighborhood $B=D \cap\left(\dot{p}_{a}>1-n^{-1}\right)$, the difference between

13. $2 \mathrm{a} \quad u=\dot{G}_{\alpha} f$

$$
\begin{aligned}
& =\dot{E} \cdot\left[\int_{0}^{\dot{\mathrm{i}} \partial D} e^{-\alpha t} f(\dot{x}) d t+e^{-\alpha \dot{\mathrm{n}} \partial \partial p} \int_{0}^{+\infty} e^{-\alpha t} f\left(\dot{x}\left(t, \dot{w}_{\dot{\mathrm{in}} \partial D}^{+}\right)\right) d t\right]
\end{aligned}
$$

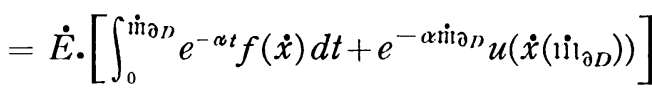

and the harmonic (and hence continuous) function 
13. $2 \mathrm{~b} \quad h=\dot{E} \cdot\left[u\left(\dot{x}\left(\dot{n i}_{\partial D}\right)\right)\right]$

is not greater than

13. 3 constant $\times\left(1-\dot{p}_{a}\right)<$ constant $\times n^{-1}$.

Because

13. $4 \quad \dot{G}_{\alpha}-\dot{G}_{\beta}+(\alpha-\beta) \dot{G}_{\alpha} \dot{G}_{\beta}=0 \quad \alpha, \beta>0$,

it is evident that $\dot{G}_{a}$ maps our space of fine continuous functions onto some subspace $D(\dot{B})$ independent of $\alpha$ and that its null-space $\dot{G}_{\alpha}^{-1}(0)$ is likewise independent of $\alpha$. But, for fine-continuous $f \in \dot{G}_{\alpha}^{-1}(0)$,

13. $50=\lim _{\beta \uparrow+\infty} \beta \dot{G_{\beta}} f=\dot{E} \cdot\left[\lim _{\beta \uparrow+\infty} \beta \int_{0}^{+\infty} e^{-\beta t} f(\dot{x}) d t\right]=f$

according to E. B. Dynkin's description of fine neighborhoods; thus the null-space is trivial, $\dot{G}_{a}$ is invertable, and another application of 13.4 verifies that $(\xi) \equiv \alpha-\dot{G}_{\alpha}^{-1}$ acting on $D\left(\xi^{3}\right)$ is independent of $\alpha$.

(अ) is the so-called generator; it is closed in the sense that if $u_{n} \in D(\dot{B})$ and $\left(\dot{B} u_{n}=f_{n}\right.$ converge pointwise under fixed bounds to $u$ and $f \in \dot{C}\left(E^{d}\right)$, then $u \in D(\dot{B})$ and $\dot{B} u=f$.

Consider the differential operator

13. 6

$$
\begin{aligned}
& \Im u=\frac{-e^{u}(d b)}{e(d b)} \quad|b|<+\infty \\
& =0 \quad b=\infty, d \geq 3
\end{aligned}
$$

acting on the class $D(\mathfrak{Q})$ of functions $u \in \dot{C}\left(E^{d}\right)$ such that

a) inside each $D, u$ is the sum of the harmonic function $h=$ $\int h_{\partial D}(a, d b) u(b)$ and the potential $\int G_{D} d e^{u}$ of its Riesz measure $e^{u}$.

b) $\int G_{D}\left|d e^{u}\right|$ is bounded.

c) $\mathfrak{Q} u$, as described in 13.6, exists and belongs to $\dot{C}\left(E^{d}\right)$.

(S) is the closure $\overline{\mathfrak{D}}$ of $\mathfrak{Q}$ in the topology of bounded pointwise convergence as will now be explained.

Choose a fine-continuous function $0<p_{n} \leq 1$ tending to 0 at $\infty$ in the ordinary topology such that $\int G_{D} p_{n} d e$ is bounded for each bounded open $D$ and $p_{n} \uparrow 1$ as $n \uparrow+\infty$, and introduce the additive functional $\dot{\mathfrak{f}}_{n}=\int_{0}^{t} p_{n}(\dot{x}) d s(t \geq 0)$. 
$\dot{x}\left(\dot{f}_{n}^{-1}\right)$ is a diffusion with Brownian hitting probabilities and speed measure $d e_{n}=p_{n} \times d e$,

$13.7 \dot{E} \cdot\left[\min \left(t: \dot{x}\left(\dot{\mathfrak{f}}_{i 2}^{-1}\right) \in \partial D\right)\right]=\dot{E} \cdot\left[\dot{f}_{n}\left(1 i_{\partial D}\right)\right]=\int G_{D} p_{n} d e<+\infty$, and it follows from 13.2a that if $u$ is in the domain of its generator $\dot{B}_{n}$, then

13.8

$$
\begin{aligned}
& u=-\dot{E} \cdot\left[\int_{0}^{\dot{n} i \partial D}\left(\left(\dot{\Theta}_{n} u\right)(\dot{x}(t)) \dot{\hat{f}}_{n}(d t)\right]+E \cdot\left[u\left(\dot{x} m_{\partial D}\right)\right)\right] \\
& =-\int G_{D}\left(\dot{\mho}_{n} u\right) p_{n} d e+a \text { harmonic function, }
\end{aligned}
$$

which is a special case of a formula of E. B. Dynkin [6]; in brief, 13.9

$$
-e^{u}(d b)=\left(\dot{\leftrightarrow}_{n} u\right) p_{n} d e \quad u \in D\left(\dot{\leftrightarrow}_{n}\right) .
$$

Choose $u=\dot{G}_{1} f \in D(\stackrel{\leftrightarrow}{)})$, then

13. $10 u_{n} \equiv \dot{E} \cdot\left[\int_{0}^{+\infty} e^{-t} f\left(\dot{x}\left(\dot{\mathfrak{f}}_{n}^{-1}\right)\right) d t\right]$

tends pointwise under the bound $\|f\|_{\infty}$ to $u$. Because $u_{n} \in D\left(\dot{\mathscr{S}}_{n}\right)$,

$$
\mathfrak{Q} u_{n}=\frac{-e^{u}(d b)}{e(d b)}=p_{n} \dot{\mathbb{S}}_{n} u_{n}=p_{n}\left(u_{n}-f\right)
$$

satisfies all the conditions for $u_{n}$ to belong to $D(\Re)$, and, what is

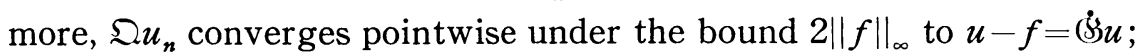
thus, $u \in D(\bar{\Omega})$ and $\overline{\mathfrak{\Omega}} u=\dot{G} u$, i.e.,

$13.12 \sqrt[\square]{ } \supset$

As to the proof of $\bar{\Omega} \subset(\dot{B}$, it is enough to show that if $u \in D(\bar{\Omega})$, then $\dot{G}_{1}(1-\bar{\Omega}) u=u$, and, for this, it is enough to deduce from $u \in D(\overline{\mathfrak{\Omega}})$ and $\overline{\mathfrak{Q}} u=u$ that $u \equiv 0$.

Given such a $u \in D(\bar{\Omega})$ with $\bar{\Omega} u=u$ and choosing $u_{n} \in D(\mathfrak{\Omega})$ so as to make $u_{n}$ and $\mathfrak{Q} u_{n}$ converge pointwise and boundedly to $u$ and $\overline{\mathfrak{Q}} \boldsymbol{u}=u$,

$$
\begin{aligned}
& u_{n}-h_{n}=-\int G_{D} \mathfrak{\Omega} u d e=-\dot{E} \cdot\left[\int_{0}^{\mathrm{n} i \partial D}\left(\Omega u_{n}\right)(\dot{x}) d s\right] \\
& h_{n}=\int h_{\partial D}(0, d b) u_{n}(b)
\end{aligned}
$$


implies

$$
\begin{aligned}
& 13.14 \dot{E} \cdot\left[\left(u_{n}-h_{n}\right)(\dot{x}(t)), t<\operatorname{li}_{\partial D}\right]-\left(u_{n}-h_{n}\right) \\
& =\dot{E} \cdot\left[\int_{0}^{t \wedge \operatorname{ni} \partial \nu}\left(\Omega u_{n}\right)(\dot{x}) d s\right] \quad t \geq 0 \text {, }
\end{aligned}
$$

which, in turn, implies

$13.15 \dot{E} \cdot\left[(u-h)(\dot{x}(t)), t<\dot{l i}_{\partial D}\right]-(u-h)$

$$
\begin{aligned}
& =\dot{E} \cdot\left[\int_{0}^{t \wedge \dot{u} \partial D}(\bar{\Omega} u)(\dot{x}) d s\right] \quad t \geq 0 \\
& h=\int h_{\partial D}(\cdot, d b) u(b)
\end{aligned}
$$

and, letting $D \uparrow R^{d}$ so as to make $h$ tend to a bounded (and hence constant) harmonic function $h(\infty)$,

13. 16a $\dot{P} \cdot\left[\mathfrak{m}_{\infty}<+\infty\right]=P \cdot\left[\mathfrak{f}\left(\mathrm{m}_{\infty}\right)<+\infty\right]=0 \quad d=2$

13.16b $u(\infty)=h(\infty)=0 \quad d \geq 3$

implies

$13.17 \dot{E} \cdot\left[u(\dot{x}(t)]-u=\dot{E} \cdot\left[\int_{0}^{t} u(\dot{x}) d s\right] \quad t \geq 0\right.$,

and the desired $u \equiv 0$ follows.

The Green operators leave invariant the space $C\left(E^{d}\right)$ of bounded functions continuous in the ordinary topology of $E^{d}$ if and only if, for each $D$, the mean exit time

$13.18 \dot{p}=\dot{E} \cdot\left[\dot{\mathfrak{m}}_{\partial D}\right]=\int G_{D} d e$

is continuous inside $D$ and tends to 0 on $\partial D$; in this case, the generator $(\dot{S})$ coincides with the differential operator $\mathfrak{a}$ acting on the class of functions $u \in C\left(E^{d}\right)$ such that $\Omega u \in C\left(E^{d}\right)$; the reader will easily supply the details of the proof.

Here is an example in which the Green operators do not map $C\left(E^{d}\right)$ into itself.

Choose $d=3$ and $e=f \times d b$, where $f=1+\sum_{n=1} f_{n}$ and the $f_{n}$ are the indicators of little non-overlapping open balls $D_{n}$ converging to as $n \uparrow+\infty$ but not covering 0 itself and so small that 
13. $19 P_{0}\left[\Pi_{\partial D}<-\infty\right]<2^{-n} \quad n \geq 1$.

Because of the first Borel-Cantelli lemma, $p \equiv P \cdot\left[\mathrm{m}_{\partial D_{n}}<+\infty\right.$, i.o. $]$ $=0$ at the origin, and it is also clear that $p \equiv 0$ on the rest of $R^{3}$. But then $\mathrm{f}=\int_{0}^{t} f(x(s)) d s$ is a continuous additive functional, $\mathrm{f}(s)<\mathrm{f}(t)(s<t)$, and $x\left(\mathrm{f}^{-1}\right)$ is a diffusion with Brownian hitting probabilities and speed measure $e$.

Given a neighborhood $D$ of 0 ,

$$
\begin{aligned}
& E_{0}\left[\min \left(t: x\left(\mathfrak{f}^{-1}\right) \in \partial D\right)\right] \\
& =E_{0}\left[f\left(\mathrm{~m}_{\partial D}\right)\right] \\
& \geq \sum_{n=1} E_{0}\left[\int_{0}^{\mathfrak{m}_{\partial D}} f_{n}(x(s)) d s\right] \\
& =\sum_{n=1} \int_{D_{n}} G_{D}(0, b) d b / \text { volume }\left(D_{n}\right) \\
& =+\infty .
\end{aligned}
$$

But, as E. B. Dynkin [6] has pointed out, this cannot happen for all small neighborhoods if the Green operators map $C\left(E^{d}\right)$ into itself.

\section{DISCONTINUOUS ADDITIVE FUNCTIONALS.}

V. A. Volkonskii [16] has studied discontinuous additive functionals; in the present Brownian case their structure is very simple.

A functional $\mathrm{t}$ of the Brownian path which satisfies

14.1 $\mathrm{t}(t, w)$ is measurable $\boldsymbol{B}_{t}$ for each $t \geq 0$.

14. $2 \quad 0 \leq \mathrm{t}<+\infty$

14. $3 \mathrm{t}(t-)=\mathrm{t}(t)$

14. $4 \mathrm{t}(t)=\mathrm{t}(s)+\mathrm{t}\left(t-s, w_{s}^{+}\right) \quad t \geq s$

is the sum of a continuous additive functional $\mathrm{f}$ and a discontinuous additive functional i with

$14.5 \quad P .[\mathrm{i}(t)=\mathrm{j}(0+), t>0]=1$

14. 6a $P \cdot[\mathrm{i}(0+)>0]=0$ or 1

14. $6 \mathrm{~b} \quad C(E)=0$, where $E$ is the set of points at which 
$P \cdot[\mathrm{i}(0+)>0]=1$ and $C$ is the Newtonian (logarithmic) capacity in $d \geq 3(=2)$ dimensions. ${ }^{9}$

Consider the (discontinuous) additive functional $\mathrm{i}_{n}(t)=$ the sum of the jumps of $\mathrm{t}$ of magnitude $\geq n^{-1}$ taking place before time $t$, note that $\left.\dot{\mathrm{i}}_{n}(0+)>0\right) \in \boldsymbol{B}_{0+}$ so that $P \cdot\left[\dot{\mathrm{t}}_{n}(0+)>0\right]=0$ or 1 according to Blumenthal's 01 law, let $E_{n}$ be the (Borel) set on which $P .\left[\dot{\mathrm{t}}_{n}(0+)>0\right]=1$, and introduce the least positive jumping time mof $\mathrm{i}_{n}$.

If $P \cdot(\mathfrak{m}<+\infty)>0$ at some point, then

$14.70<P .\left[0<\mathrm{m}<+\infty, \dot{\mathrm{j}}_{n}(\mathrm{~m})<\mathrm{i}_{n}(\mathrm{~m}+)\right]$

$$
=P \cdot\left[0<\mathrm{m}<+\infty, x(\mathfrak{m}) \in E_{n}\right] ;
$$

this implies $C\left(E_{n}\right)>0{ }^{10}$, and it follows that $E_{n}$ contains a subcompact $A$ of positive capacity, having a (regular) point at which $P .[x(t) \in A$, i.o., $t \downarrow 0]=1 .^{11} \quad$ But then $P \cdot\left[\mathrm{i}_{n}(t) \equiv+\infty, t>0\right]=1$ at that point, contradicting $\mathrm{i}_{n} \leq \mathrm{t}<+\infty$, and it follows that

14. 8a $\quad P \cdot\left[\dot{\mathrm{t}}_{n}(t) \equiv \mathrm{j}_{n}(0+), t>0\right] \equiv 1$

14. $8 \mathrm{~b} \quad C\left(E_{n}\right)=0$.

The rest is clear: $\mathrm{i}=\lim _{\uparrow \uparrow+\infty} \dot{\mathrm{j}}_{n}$ satisfies 14.5 , the remainder $\mathrm{f}=\mathrm{t}-\mathrm{i}$ is a continuous additive functional, 14. $6 \mathrm{a}$ is immediate from Blumenthal's 01 law, and $C(E)=\lim _{n \uparrow+\infty} C\left(E_{n}\right)=0$.

Massachusetts Institute of Technology

Kyushu University

9 G. Choquet [3] found that if $E \subset R^{d}$ is Borel, then inf $C(B): B$ open, $B \supset E$

$=\sup C(A): A$ compact, $A \subset E$;

this common value is the capacity of $E$.

${ }^{10} P .[x(t) \in E$ at some positive time $]$ is positive or $\equiv 0$ according as $C(E)>0$ or not ; see, for example, G. Hunt [9].

11 See O. D. Kellogg [11] for the classical significance of regular points and J. Doob [5] for the probabilistic interpretation. 


\section{REFERENCES}

[1] R. Blumenthal : An extended Markov property. Trans. Am. Math. Soc. 85 (1957), 52-72.

[2] H. Cartan: Théorie générale du balayage en potentiel newtonien. Ann. Univ. Grenoble 22 (1946), 221-280.

[3] G. Choquet: Theory of capacities. Ann. Inst. Fourier Grenoble, 5 (1953-1956), 131-295.

[4] J. Doob: Stochastic Processes. J. Wiley and Sons. New York (1953).

[5] : Semimartingales and subharmonic functions. Trans. Am. Math. Soc. 77 (1954), 86-121.

[6] E. B. Dynkin: Infinitesimal operators of Markov processes. Teor. Veroyat. i Primenen. 1 (1956), 38-60.

[7] — : Natural topology and excessive functions connected with a Markov process. Doklady Akademii Nayk CCCP, 127 (1959), 17-19.

[8] W. Feller: The general diffusion operator and positivity preserving semi-groups in one dimension. Ann. Math. 60 (1954), 417-436.

[9] G. Hunt: Some theorems concerning Brownian motion. Trans. Am. Math. Soc. 81 (1956), 294-319.

[10] : Markov processes and potentials (1). Illinois J. Math. 1 (1957), 44-93.

[11] O. D. Kellogg: Foundations of Potential Theory. J. Springer. Berlin (1929).

[12] K. Itô and H. P. McKean, Jr.: Potentials and the random walk. Illinois J. Math. 4 (1960), 119-132.

[13] : Diffusion. to appear.

[14] F. Riesz: Sur les fonctions subharmoniques et leur rapport a la théorie du potentiel. Acta Math. 48 (1926), 329-343.

[15] A. Volkonskiri: Random substitution of time in strong Markov process. Teor. Veroyat. i Primenen. 3 (1958), 332-350.

[16] : Additive functionals of Markov processes. Doklady Akademii Nayk CCCP. 127 (1959), 735-738.

Note added in proof. The authors came to know that A. D. Ventsel' [19] obtained almost the same result as in sect. 3-5 of the present paper and that A. Meyer [18] studied the same problem for a more general class of Markov processes. As for signed additive functionals, E. B. Dynkin [17] constructed them using stochastic integrals in case of a Brownian motion.

[17] E. B. Dynkin: Additive functionals of a Wiener process determined by stochastic integrals. Teor. Veroyat. i Primenen. 5 (1960), 441-452.

[18] A. Meyer: Representations integrales des potentiels. C. R. Paris 251 (1960), 22 79-2280.

[19] A. D. Ventsel' : Non-negative additive functionals of Markov processes. Doklady Akademii Nayk CCCP 137 (1961), 17-20 